\title{
ARTICLE
}

Infectious medicine, virology

\section{The Janus kinase $1 / 2$ inhibitor ruxolitinib in COVID-19 with severe systemic hyperinflammation}

\author{
F. La Rosée $\mathbb{1}^{1} \cdot$ H. C. Bremer ${ }^{2} \cdot$ I. Gehrke ${ }^{3}$ A. Kehr ${ }^{3} \cdot$ A. Hochhaus ${ }^{4} \cdot$ S. Birndt $\mathbb{D}^{4} \cdot$ M. Fellhauer ${ }^{5} \cdot$ M. Henkes ${ }^{6}$. \\ B. Kumle ${ }^{7}$. S. G. Russo $0^{8,9} \cdot$ P. La Rosée $\mathbb{1}^{6,10}$
}

Received: 8 May 2020 / Revised: 14 May 2020 / Accepted: 22 May 2020 / Published online: 9 June 2020

(c) The Author(s) 2020. This article is published with open access

\begin{abstract}
A subgroup of patients with severe COVID-19 suffers from progression to acute respiratory distress syndrome and multiorgan failure. These patients present with progressive hyperinflammation governed by proinflammatory cytokines. An interdisciplinary COVID-19 work flow was established to detect patients with imminent or full blown hyperinflammation. Using a newly developed COVID-19 Inflammation Score (CIS), patients were prospectively stratified for targeted inhibition of cytokine signalling by the Janus Kinase $1 / 2$ inhibitor ruxolitinib (Rux). Patients were treated with efficacy/toxicity guided step up dosing up to 14 days. Retrospective analysis of CIS reduction and clinical outcome was performed. Out of 105 patients treated between March 30th and April 15th, 2020, 14 patients with a CIS $\geq 10$ out of 16 points received Rux over a median of 9 days with a median cumulative dose of $135 \mathrm{mg}$. A total of 12/14 patients achieved significant reduction of CIS by $\geq 25 \%$ on day 7 with sustained clinical improvement in 11/14 patients without short term red flag warnings of Ruxinduced toxicity. Rux treatment for COVID-19 in patients with hyperinflammation is shown to be safe with signals of efficacy in this pilot case series for CRS-intervention to prevent or overcome multiorgan failure. A multicenter phase-II clinical trial has been initiated (NCT04338958).
\end{abstract}

\section{Introduction}

The novel coronavirus (SARS-CoV-2) pandemic is a global health crisis. A $1-5 \%$ mortality rate affecting particularly comorbid patients is currently observed [1]. The disease has been designated COVID-19, an acronym for "coronavirus

These authors contributed equally: F. La Rosée, H.C. Bremer

P. La Rosée

paul.1arosee@sbk-vs.de

1 Medizinische Fakultät Carl Gustav Carus, Technische Universität Dresden, Dresden, Germany

2 Lungenzentrum Donaueschingen, Schwarzwald-Baar-Klinikum, Villingen-Schwenningen, Germany

3 Klinik für Innere Medizin IV, Schwarzwald-Baar-Klinikum, Villingen-Schwenningen, Germany

4 Klinik für Innere Medizin II, Universitätsklinikum Jena, Jena, Germany

5 Apotheke/Institut für Klinische Pharmazie, Schwarzwald-BaarKlinikum, Villingen-Schwenningen, Germany disease 2019". To date, efficacy of antiviral drugs explored in COVID-19 awaits confirmation [2, 3].

While most people with COVID-19 develop mild or uncomplicated illness, $\sim 14 \%$ develop severe disease requiring hospitalization and oxygen support and 5\% require admission to an intensive care unit. In severe cases, COVID-19 can be complicated by acute respiratory distress syndrome (ARDS), sepsis, and/or multiorgan failure (MOF) [4]. Recent multivariable analysis confirmed older age, higher Sequential Organ Failure Assessment (SOFA) score

6 Klinik für Innere Medizin II, Hämatologie, Onkologie, Immunologie, Infektiologie und Palliativmedizin, SchwarzwaldBaar-Klinikum, Villingen-Schwenningen, Germany

7 Klinik für Akut- und Notfallmedizin, Schwarzwald-BaarKlinikum, Villingen-Schwenningen, Germany

8 Klinik für Anästhesiologie, Intensiv-, Notfall- und Schmerzmedizin, Villingen-Schwenningen, Germany

9 Medizinische Fakultät, Universität Göttingen, Göttingen, and Fakultät für Gesundheit, Universität Witten/Herdecke, Witten, Germany

10 Medizinische Fakultät der Friedrich-Schiller-Universität Jena, Universitätsklinikum Jena, Jena, Germany 
and D-Dimer $>1 \mu \mathrm{g} / \mathrm{L}$ on admission associated with higher mortality [5].

Severe and critically affected patients develop bilateral viral pneumonia, which is categorized as hypersensitivity pneumonitis [6]. Autopsy lung tissue shows diffuse infiltration of hyperactivated T-cells, as does T-cell typing in the peripheral blood [7]. Chinese COVID-19 series report hyperinflammation governed by pro-inflammatory cytokines in particular in patients with dismal outcome indicating a significant role of cytokine release for tissue damage and multiorgan failure [4, 8, 9]. Management of COVID-19 patients requires hospitals to setup COVID-19 specific infrastructure to prevent uncontrolled transmissions, establish high-end multidisciplinary knowledge teams to provide a continuum of care from emergency room to intensive care unit (ICU) and into weaning and rehabilitation facilities.

Ruxolitinib (Rux; Jakavi ${ }^{\circledR}$ ) is a potent and selective inhibitor of Janus kinases (JAK) 1 and 2, with modest to marked selectivity against tyrosine kinase (TYK)2 and JAK3, respectively. Rux is currently approved in the European Union (EU) for the treatment of primary myelofibrosis (PMF), post-polycythemia vera (PV) or post-essential thrombocythemia myelofibrosis and for the treatment of adult patients with PV [10]. Key to Rux efficacy is its broad anti-inflammatory activity against the myeloproliferative neoplasm (MPN) inherent cytokine storm with proinflammatory IL-1, IL-6, IL-8, IL-12, TNF- $\alpha$, IFN $\gamma$, VEGF, TGF $\beta$, FGF, PDGF, GM-CSF, and G-CSF cytokines/growth factors [11]. Rux is highly effective in off-label indications, where cytokine release plays a central role for pathogenesis: Graft versus host disease (GvHD) and hemophagocytic lymphohistiocytosis (HLH) [12-15]. Rux doses ranging from $5 \mathrm{mg}$ bid (GvHD) up to $25 \mathrm{mg}$ bid (HLH) were successfully used without signs of overt toxicity. As both conditions go along with a significant risk of viral or bacterial reactivation, safe immunomodulation without safety signals is of particular interest in light of unknown mechanisms of SARS-CoV-2 viral clearance [16-18].

Many patients with severe respiratory disease due to COVID-19 have features consistent with cytokine release syndrome (CRS) [19, 20]. Due to increased activation of the JAK/STAT pathway, it is postulated that JAK-inhibitors might have a useful role in treating these patients [21, 22].

\section{Methods}

\section{Study design}

This is a monocentric retrospective chart analysis on consecutive patients admitted to the Schwarzwald-Baar-Klinikum Villingen-Schwenningen, Germany, with severe
Table 1 COVID hyperinflammation score ( $\geq 10$ of 16 threshold for inclusion).

\begin{tabular}{ll}
\hline Points & \\
\hline Chest-X-ray/Chest-CT consistent w/ & $\mathbf{3}$ \\
hypersensitivity pneumonitis & \\
CRP $>20 \times$ ULN & $\mathbf{2}$ \\
Ferritin $>2 \times$ ULN & $\mathbf{2}$ \\
Triglycerides $>1.5 \times$ ULN & $\mathbf{1}$ \\
IL- $6>3 \times$ ULN & $\mathbf{1}$ \\
Fibrinogen $>$ ULN & $\mathbf{1}$ \\
Leukocytes $>$ ULN & $\mathbf{1}$ \\
Lymphopenia $<1.1 / n L$ & $\mathbf{2}$ \\
Fever $>38.5^{\circ}$ C & $\mathbf{2}$ \\
Coagulation disorder & $\mathbf{1}$ \\
- DIC $($ D-Dimer $>$ ULN) & \\
- PTT $>$ ULN &
\end{tabular}

ULN Upper limit of normal, DIC Disseminated Intravascular Coagulation, PTT Partial thromboplastin time.

COVID-19 and a multidisciplinary board decision on specific medical treatment. Assessment of systemic inflammation was done using a trial specific newly developed clinical inflammation score, named COVID Inflammation Score (CIS) (Table 1). The score was developed through integration of published patient characteristics from the Chinese case series [5, 23, 24]. Patients achieving the threshold score value of $\geq 10$ (out of max. 16 score points) without clinical signs of sepsis (procalcitonin (PCT) negative, no uncontrolled active infection) were deemed at high risk for systemic inflammation based on cytokine release and evaluable for Rux treatment. First patient treated was March 30th, 2020. Date of last treatment initiation was April 15th, 2020 with cut-off for follow-up on April 21st, 2020 (patient $\# 14$, day 7). Severity was defined if any of the following conditions was met: (1) respiratory rate $\geq 30$ breaths/min; (2) $\mathrm{SpO}_{2} \leq 93 \%$ while breathing ambient air; (3) $\mathrm{PaO}_{2} /$ $\mathrm{FiO}_{2} \leq 300 \mathrm{mmHg}$. Critical COVID-19 was diagnosed if any of (1) respiratory failure requiring mechanical ventilation, (2) shock, (3) combined with other organ failure requiring admission to ICU occurred.

\section{Ruxolitinib treatment}

Rux was provided by the hospital pharmacy as $15 \mathrm{mg}$ tablets. Based on available prescription data on Rux and devoid of publicly available data on Rux in COVID-19, we decided on an intermediate dose between published trial results in GvHD (5 mg bid) and hemophagocytic lymphohistiocytosis (15 mg bid) and started treatment with $7.5 \mathrm{mg}$ bid $[12,25]$. Daily follow-up of efficacy and toxicity guided dosage with stepwise dose increase (15mg-0-7.5 mg; $15 \mathrm{mg}$ $0-15 \mathrm{mg}$ ) at days 3,5 , or 7 by COVID-board decision was in 
place. Extended treatment duration in patients with clinical benefit and careful benefit-risk assessment was decided individually. Patients with active infections, severe hepatic impairment prior to systemic inflammation and underlying comorbidity with inherent survival probability $<6$ months were excluded. Recommendations for supportive and antiviral treatment were taken from the national COVID-19 guidelines [26].

\section{Efficacy and toxicity assessment}

Efficacy was defined as achievement of $25 \%$ reduction in the CIS on day 7 compared to baseline. Radiologic response was taken from the X-ray/CT reports: "Deteriorated" compared with baseline was scored "3", unchanged "2", improved "1", "resolved" was scored "0". Ferritin response received gradual scoring for response assessment according to percent change of serum concentration compared to baseline: $>20 \%$ increase scored " 2 " (progression), +/- 20\% scored " 1 " (unchanged), and $>20 \%$ decrease compared to baseline was scored 0 (response). Response for the reminder parameters was defined binary, i.e. reduction below the defined inflammation level was scored " 0 ". The clinical course of patients was assessed by the 7 point ordinal WHO scale at baseline, day 7 and day 15 as proposed by the WHO R\&D blueprint (https://www.who.int/blueprint/priority-disea ses/key-action/novel-coronavirus/en/). In adddition, the NEWS2-score to assess ICU-parameters for respiratory state and vital signs was assessed along days 0, 5, 7, and 15 (https://www.rcplondon.ac.uk/news/news2-anddeterioration-COVID-19). Toxicity was assessed looking at adverse events of special interest as provided in the Jakavi $^{\circledR}$ prescription information (hematologic toxicity, liver toxicity) according to common toxicity criteria of adverse events version 5.0 (CTCAE 5.0).

\section{Structured COVID-19 care algorithm (COVID-19-SOP)}

The medical team caring for COVID-19 was structured into the respiratory/IMC/ICU core team (pneumologists/ICU care specialists), the regular care team for primary workup of patients with respiratory symptoms and fever and the immunomodulation team from Hematology/Immunology. The Schwarzwald-Baar-Klinikum (SBK) is a two campus General Academic Teaching Hospital of the University of Freiburg, allowing separation of COVID-19 patients in the Lung Center Campus Donaueschingen from the main campus (Villingen-Schwenningen). Special consideration was given COVID-19 ventilation management focusing on optimized non-invasive ventilation. This hospital wide respiratory management was developed to prevent patients from early intubation as the COVID-19-specific lung damage causes high mortality rates on invasive ventilation [27]. Patients received acetylsalicylic acid $500 \mathrm{mg}$, ascorbic acid $1000 \mathrm{mg}$, hydroxychloroquine $600 \mathrm{mg}$ bid day 1, and $200 \mathrm{mg}$ bid day 2-5, low molecular weight heparin providing therapeutic anticoagulation guided by D-Dimer levels and empiric antibiotic treatment. A defined COVID-lab sample was taken including extended coagulation parameters (PTT, D-Dimer, Fibrinogen), inflammation markers (C-reactive protein, ferritin, IL-6, sIL2-R, triglycerides) and routine emergency room lab tests including haematology. Daily COVID-19 board meetings were held with structured board reports. The CIS was calculated to aid treatment decisions regarding specific inflammation directed treatment with Rux. Short term corticosteroids at $2 \mathrm{mg} / \mathrm{kg}$ day $1-3$ prednisolone was allowed based on individual comorbidity and disease severity aspects. Patients with excessive serum IL-6 levels $>200 \mathrm{pg} / \mathrm{ml}$ and critical conditions were candidates for anti-IL-6 blockade by tocilizumab $400 \mathrm{mg}$ single dose (patients \#2, \#13) according to recently discussed data on the WHO COVID-19 global literature database (https:// search.bvsalud.org/global-literature-on-novel-coronavirus2019-ncov/; accessed April 24th, 2020).

\section{Data analysis}

Data were retrieved from patient charts and the electronic hospital information System Orbis (AGFA Health Care $\mathrm{GmbH}$, Bonn, Germany). Systematic data acquisition for descriptive analysis was done using MS Excel (Microsoft, Redmond, WA, USA). Significance testing for CIS reduction as surrogate endpoint was done using Wilcoxon test for related samples using calculator on https://www.socscista tistics.com (two-sided, significance level 0.05).

\section{Results}

\section{Patient characteristics}

From March 30th to April 15th, 2020, $n=105$ patients were hospitalized for COVID-19 treatment (Fig. 1). Patient characteristics are shown in Table 2 with a male predominance in the $\mathrm{CIS} \geq 10$ hyperinflammatory patient cohort. COVID-19 was confirmed via SARS-CoV-2-PCR positivity. A total of $66 / 105(63 \%)$ patients stratified to standard of care (SOC) treatment recovered without further intervention. A total of $12 / 105(11 \%)$ patients with primary stratification to palliative care died $(n=7)$ or were still hospitalized at data cut-off $(n=5)$. A total of 27/105 patients deteriorated on SOC and entered COVID-19 interdisciplinary board assessment after determination of the CIS (Table 1). Patients at high risk for hyperinflammation according to CIS and stratified to Rux treatment 


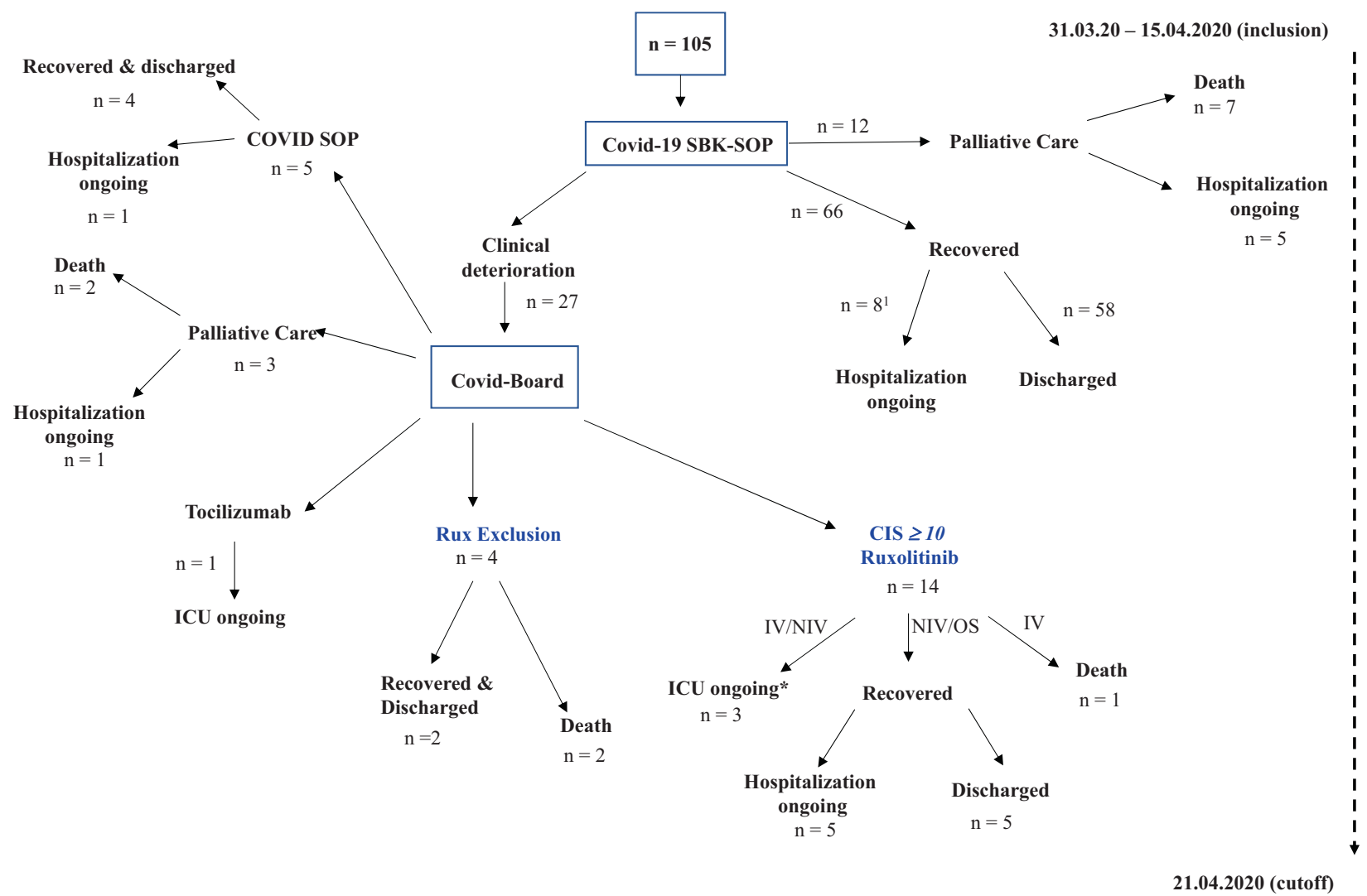

Fig. 1 COVID-19 patient flow at Schwarzwald-Baar-Klinikum during March 31th and April 21st 2020. IV invasive ventilation, NIV non-invasive ventilation, OS oxygen support, $*_{n}^{*}=1$ Rux after Tocilizumab. ${ }^{1}$ Two patients started Ruxolitinib after data cutoff and recovered.

were 66 (55-81) years old with a male predominance (11/ 14) (Table 2). Ten out of 14 patients presented with fever $>38.5^{\circ} \mathrm{C}$, the majority was on non-invasive ventilation (10/ 14 ), two were on oxygen support (3-4 1/min), one on highflow oxygen $(20 \mathrm{l} / \mathrm{min}$ ) and one patient was admitted via inter-hospital transfer on invasive ventilation $\left(\mathrm{FiO}_{2} 90 \%\right)$. All patients presented with radiologic signs consistent with bilateral COVID-19 pneumonitis or COVID-19 associated ARDS. Arterial hypertension (11/14), smoking (9/14), hyperlipidemia (7/14), diabetes mellitus (5/14), and preexisting lung disease (5/14) were the leading comorbidities. One patient was on postsurgical care after curative treatment for lung cancer with intrahospital CoV-2-infection and another patient on immunosuppression for vasculitis. A total of 5/14 patients presented with a respiratory rate $>25$ / min. The median NEWS2-score at baseline was 8.5 (4-16) indicating severe or critical COVID-19 in all patients stratified to Rux (Table 2).

\section{COVID inflammation score (CIS) stratified medical treatment}

Median days from onset of first symptoms to hospitalization and to Rux treatment were 9 (4-19) and 15.5 (5-24), respectively (Table 3 ). C-reactive protein (CRP) was highly elevated in the majority with 22.3 (1.6-67) fold upper limit of normal (ULN) (Table 4). Interleukin-6 (IL6) baseline values showed high variability with a median of 19 (3-282) fold ULN. Ferritin confirmed high inflammatory activity with $1585 \mathrm{ng} / \mathrm{ml}$ median elevation in conjunction with soluble interleukin-2 receptor (sIL2-R) positivity (1673 U/ml (994-4917)). In contrast, procalcitonin was normal in all but one patient. This single patient was deemed "sepsis uncertain" due to vasculitis-dependent PCT activation as consulted by the rheumatologist. Patients were not cytopenic except for anemia in individual patients (median $\mathrm{Hb} 12.9 \mathrm{~g} / \mathrm{dl}(8.6-15.9)$ ). Liver function was nearly normal at baseline with median alanine aminotransferase (ALT) and aspartate aminotransferase (AST) values sharply above ULN (Table 4).

The median CIS of patients stratified to CRS-targeted treatment was 12 (10-14) at baseline (Fig. 1). Treatment with starting dose of $7.5 \mathrm{mg}$ Rux bid led to marked clinical control within days (patients \#1, 3, 4, 6, 8, 9, 10, 11, 14) (Fig. 2). CIS reduction by $25 \%$ was achieved on day 5 and day 7 with a decline by $42 \%$ and $58 \%$, respectively (Fig. 1, Table 5).

Three patients with insufficient response were dose escalated to $15 \mathrm{mg}$ bid (day 16, patient \#7), and 15mg-0-7.5 
Table 2 Baseline demographics and clinical characteristics.

\begin{tabular}{|c|c|c|c|c|}
\hline Characteristics (total cohort) & \multicolumn{4}{|l|}{ Total $(N=105)$} \\
\hline Age, median (IQR)-years & \multicolumn{4}{|l|}{$65(32-95)$} \\
\hline Male sex-no. $(\%)$ & \multicolumn{4}{|l|}{$58(55)$} \\
\hline \multicolumn{5}{|l|}{ Coexisting conditions-no. $(\%)$} \\
\hline Diabetes mellitus & \multicolumn{4}{|l|}{$28(27)$} \\
\hline Arterial hypertension & \multicolumn{4}{|l|}{$67(64)$} \\
\hline Hyperlipidemia & \multicolumn{4}{|l|}{$21(20)$} \\
\hline Cerebrovascular disease & \multicolumn{4}{|l|}{$5(5)$} \\
\hline Cardiovascular disease & \multicolumn{4}{|l|}{$36(34)$} \\
\hline Lung disease & \multicolumn{4}{|l|}{$34(32)$} \\
\hline Malignant disease & \multicolumn{4}{|l|}{$16(15)$} \\
\hline Immunosuppression & \multicolumn{4}{|l|}{$1(1)$} \\
\hline Smoker/Ex-smoker & \multicolumn{4}{|l|}{$28(27)$} \\
\hline Characteristics (Rux-cohort) & Total $(N=14)$ & $\begin{array}{l}\text { Invasive } \\
\text { ventilation }(N=1)\end{array}$ & $\begin{array}{l}\text { Non-invasive } \\
\text { ventilation }(N=10)\end{array}$ & $\begin{array}{l}\text { Oxygen support } \\
(N=3)\end{array}$ \\
\hline Age, median (IQR)-years & $66(55-81)$ & 65 & $65(55-81)$ & $68(64-70)$ \\
\hline Male sex-no. $(\%)$ & $11(79)$ & 1 & $7(70)$ & $3(100)$ \\
\hline \multicolumn{5}{|l|}{ Coexisting conditions-no. $(\%)$} \\
\hline Diabetes mellitus & $5(36)$ & 1 & $4(40)$ & $0(0)$ \\
\hline Arterial hypertension & $11(79)$ & 1 & $7(70)$ & $3(100)$ \\
\hline Hyperlipidaemia & $7(50)$ & 1 & $3(30)$ & $3(100)$ \\
\hline Cerebrovascular disease & $2(14)$ & 0 & $0(0)$ & $2(67)$ \\
\hline Cardiovascular disease & $6(43)$ & 1 & $3(30)$ & $2(67)$ \\
\hline Lung disease & $5(36)$ & 0 & $4(50)$ & $1(33)$ \\
\hline Malignant disease & $1(7)$ & 0 & $0(0)$ & $1(33)$ \\
\hline Immunosuppression & $1(7)$ & 0 & $1(10)$ & $0(0)$ \\
\hline Smoker/Ex-smoker & $9(64)$ & 1 & $6(60)$ & $2(67)$ \\
\hline Fever $\geq 38.5^{\circ} \mathrm{C}-$ no. $(\%)$ & $10(71)$ & 0 & $8(80)$ & $2(67)$ \\
\hline $\begin{array}{l}\text { Respiratory rate } \geq 25 / \mathrm{min}- \\
\text { no. }(\%)\end{array}$ & $5(36)$ & 1 & $2(20)$ & $2(67)$ \\
\hline $\begin{array}{l}\text { Systolic blood pressure } \\
<90 \mathrm{mmHg}-\text { no. }(\%)\end{array}$ & $0(0)$ & 0 & $0(0)$ & $0(0)$ \\
\hline \multicolumn{5}{|c|}{ Oxygen-support category - no. (\%) } \\
\hline Invasive ventilation & $1(7)$ & 1 & - & - \\
\hline Non-invasive ventilation & $10(71)$ & - & $10(100)^{\mathrm{a}}$ & - \\
\hline High-flow oxygen support & $1(7)$ & - & - & $1(33)$ \\
\hline Low-flow oxygen support & $2(14)$ & - & - & $2(67)$ \\
\hline \multicolumn{3}{|c|}{ NEWS2 score at baseline, median (IQR) } & $8.5(4-16)$ & \\
\hline \multicolumn{5}{|c|}{ Seven-point ordinal scale at baseline } \\
\hline \multicolumn{3}{|c|}{ 4: Hospitalization, requiring supplemental oxygen-no. $(\%)$} & \multicolumn{2}{|l|}{$2(14)$} \\
\hline \multicolumn{3}{|c|}{ 5: Hospitalization, requiring HFNC or non-invasive mechanical ventilation-no. (\%) } & \multicolumn{2}{|l|}{$11(79)$} \\
\hline \multicolumn{3}{|c|}{ 6: Hospitalization, requiring ECMO, invasive mechanical ventilation, or both-no. (\%) } & \multicolumn{2}{|l|}{$1(7)$} \\
\hline
\end{tabular}

$H F N C$ high-flow nasal cannula, ECMO extracorporeal membrane oxygenation.

mg (patients \#2 and \#5). Patients \#7 and \#5 finally were consented to treatment limitation due to progressing multiorgan failure. Patient \#7 (65 y/o) entered specific Rux treatment after emergency interhospital transfer at high level invasive ventilation $\left(\mathrm{FiO}_{2} \quad 0.9\right)$ and was significantly comorbid (diabetes mellitus, arterial hypertension, coronary heart disease, smoking, hyperlipidemia). Patient \#5 (70 y/o) suffered from significant pre-existing comorbidities (chronic obstructive lung disease, smoking, obesity, arterial hypertension). Patient \#12 with elevated PCT at first 
COVID-board presentation (ongoing treatment of polyangiitis with pulmonary and renal affection; on azathioprine and chronic dialysis) was observed and treated according to SOC for sepsis. After rheumatology consult, the diagnosis

Table 3 Patient clinical assessment and treatments received.

\begin{tabular}{lc}
\hline & Total $(N=14)$ \\
\hline Treatment since hospitalization, no. (\%) & $3(21)$ \\
Invasive ventilation & $13(93)$ \\
Non-invasive ventilation & $1(7)$ \\
Renal-replacement therapy & $12(86)$ \\
Antibiotic agent & $13(93)$ \\
Hydroxychloroquine & $4(29)$ \\
Vasopressors & $2(14)$ \\
Tocilizumab & $11(79)$ \\
Glucocorticoid therapy & $3(3-15)$ \\
Days of glucocorticoid therapy, median (IQR) & \\
Ruxolitinib dosage and therapy length & $135(52.5-285)$ \\
Cumulative dosage, median (IQR)—mg & $9(5-17)$ \\
Length of treatment, median (IQR)—days & $15.5(5-24)$ \\
Days from illness onset to Ruxolitinib treatment, \\
median (IQR)
\end{tabular}

sepsis without proven bloodstream infection was weighed against vasculitis-associated PCT "false-positivity". Single dose Rux $7.5 \mathrm{mg}$ was initiated but treatment was limited due to progressing multiorgan failure. Patient \#5 died on palliative care day 17 after Rux onset.

Patient \#2 showed marked clinical and inflammatory response on dose escalated Rux and was weaned to $\mathrm{O}_{2}$ supply (Fig. 3).

As the majority of patients was treated with short course of $2 \mathrm{mg} / \mathrm{kg}$ prednisolone over three days, we asked, whether Rux treatment can demonstrate CIS-inhibition with clinical response in the absence of corticosteroids. Four patients with a board-decision for contraindications against CS (patients \#2, \#9, \#14) or with a CS-free treatment window of 8 days (patient \#11) were put on Rux with marked clinical and CIS-response. Figure 2 demonstrates laboratory response for CRP and IL-6 with variable dynamics of ferritin and lymphocytes in all CS-free treatment episodes, indicating CS-independent anti-inflammatory activity of Rux in COVID-19 induced CRS.

\section{Toxicity and safety}

Ruxolitinib has potential for drug induced liver damage, impairment of hematopoiesis and uncertainty with regards to infection control due to reports of viral/bacterial reactivation in patients with MPN [28]. As demonstrated in

Table 4 Laboratory assessment.

\begin{tabular}{|c|c|c|c|c|c|}
\hline Toxicity and Laboratory values_-median (IQR) & Normal range & Baseline & Day 5 & Day 7 & Day 15 \\
\hline Serum Creatinine, mg/dl & $0.5-1.1$ & $1.01(0.74-6.57)$ & $0.89(0.64-4.3)$ & $0.82(0.51-6.43)$ & $0.92(0.55-1.13)$ \\
\hline total Bilirubin, $\mathrm{mg} / \mathrm{dl}$ & $0.2-1.1$ & $0.5(0.1-0.9)$ & $0.5(0.1-2.2)$ & $0.45(0.1-1.6)$ & $0.65(0.5-0.8)$ \\
\hline AST, U/1 & $<50$ & $54(22-150)$ & $66.5(26-196)$ & $56.5(27-176)$ & $37(23-116)$ \\
\hline ALT, U/l & $<50$ & $49.5(21-157)$ & $109(27-299)$ & $100(34-415)$ & $83(36-221)$ \\
\hline Triglycerides, mg/dl & $<150$ & $179(116-512)$ & $258 \cdot 5(114-432)$ & $252(171-311)$ & $158.5(95-263)$ \\
\hline Triglycerides, x ULN & & $1.2(0.8-3.4)$ & $1.75(0.8-2.9)$ & $1.7(1.1-2.1)$ & $1(0.6-1.8)$ \\
\hline $\mathrm{LDH}, \mathrm{U} / \mathrm{l}$ & $<250$ & $456.5(283-990)$ & $355.5(207-790)$ & 333 (199-737) & $444(338-777)$ \\
\hline Ferritin, ng/ml & $15-400$ & $1585(498-6931)$ & $1678.5(306-3891)$ & $1532(274-3483)$ & $1488(1006-2958)$ \\
\hline Ferritin, x ULN & & $4(1.3-43.3)$ & $4.2(1.8-24.3)$ & $3.8(1.3-21.7)$ & $3.7(2.5-18.5)$ \\
\hline $\mathrm{PTT}>\mathrm{ULN}$ & & $0.9(0.7-1.5)$ & $0.9(0.7-1.8)$ & $0.9(0.7-1.1)$ & $0.8(0.7-1.1)$ \\
\hline Fibrinogen, $\mathrm{x}$ ULN & & $1.3(0.7-2.5)$ & $1.5(0.7-2)$ & $1.6(0.9-2)$ & $1.3(0.8-1.6)$ \\
\hline D-Dimer, x ULN & & $3.5(1.1-21.8)$ & $2.3(1.1-22.9)$ & $2.5(0.8-19.5)$ & $5.5(0.5-8.6)$ \\
\hline $\mathrm{C}$ reactive protein, $\mathrm{x}$ ULN & & $22.3(1.6-67)$ & $10.3(1-56.7)$ & $5.4(0.7-51.9)$ & $3.1(0.3-48.7)$ \\
\hline Procalcitonin ng/ml & $<0.5$ & $0.17(0.02-3.79)$ & $0.07(0.02-1.21)$ & $0.05(0.02-1.16)$ & $0.04(0.02-0.43)$ \\
\hline IL-6, x ULN & & $19(3.1-282.1)$ & $3.3(0.4-179.4)$ & $1.25(0.2-119.4)$ & $5.9(2.1-11.2)$ \\
\hline sIL2-R, U/ml & $158-623$ & 1673 (994-4917) & $1340.5(718-3568)$ & $1052(733-1877)$ & $778(687-1255)$ \\
\hline \multicolumn{6}{|l|}{ Hematological laboratory } \\
\hline White blood-cell count, /nl & $4-10$ & $8.3(5-17)$ & $10(4.4-15.7)$ & $8.4(3.3-17.2)$ & $8.2(3.9-20.2)$ \\
\hline White blood-cell count, x ULN & & $0.8(0.5-1.7)$ & $1(0.4-1.6)$ & $0.8(0.3-1.9)$ & $0.8(0.4-2)$ \\
\hline Platelet count, /nl & $150-450$ & $189.5(105-397)$ & $324.5(124-850)$ & 379.5 (109-937) & $301.5(223-540)$ \\
\hline Neutophil count, /nl & $1.4-6.5$ & $6.14(3.59-13.76)$ & $6.96(2.6-12.84)$ & $5.94(1.84-15.48)$ & $3.94(2.6-16.22)$ \\
\hline Lymphocyte count, /nl & $1.2-3.4$ & $0.97(0.52-2.15)$ & $1.23(0 \cdot 71-3.7)$ & $1.27(0.51-3.54)$ & $1.68(0.82-3.09)$ \\
\hline Hemoglobin, $\mathrm{g} / \mathrm{dl}$ & $12-18$ & $12.9(8.6-15.9)$ & $12.3(7.7-14.5)$ & $12.2(7.4-15.5)$ & $10.2(7.9-13.6)$ \\
\hline
\end{tabular}


Fig. 2 COVID inflammation score at baseline, day 5 and day 7 after Rux treatment initiation. Dots represent individual patient results. Median and IQR are provided by box plots. $* p<0.01$.

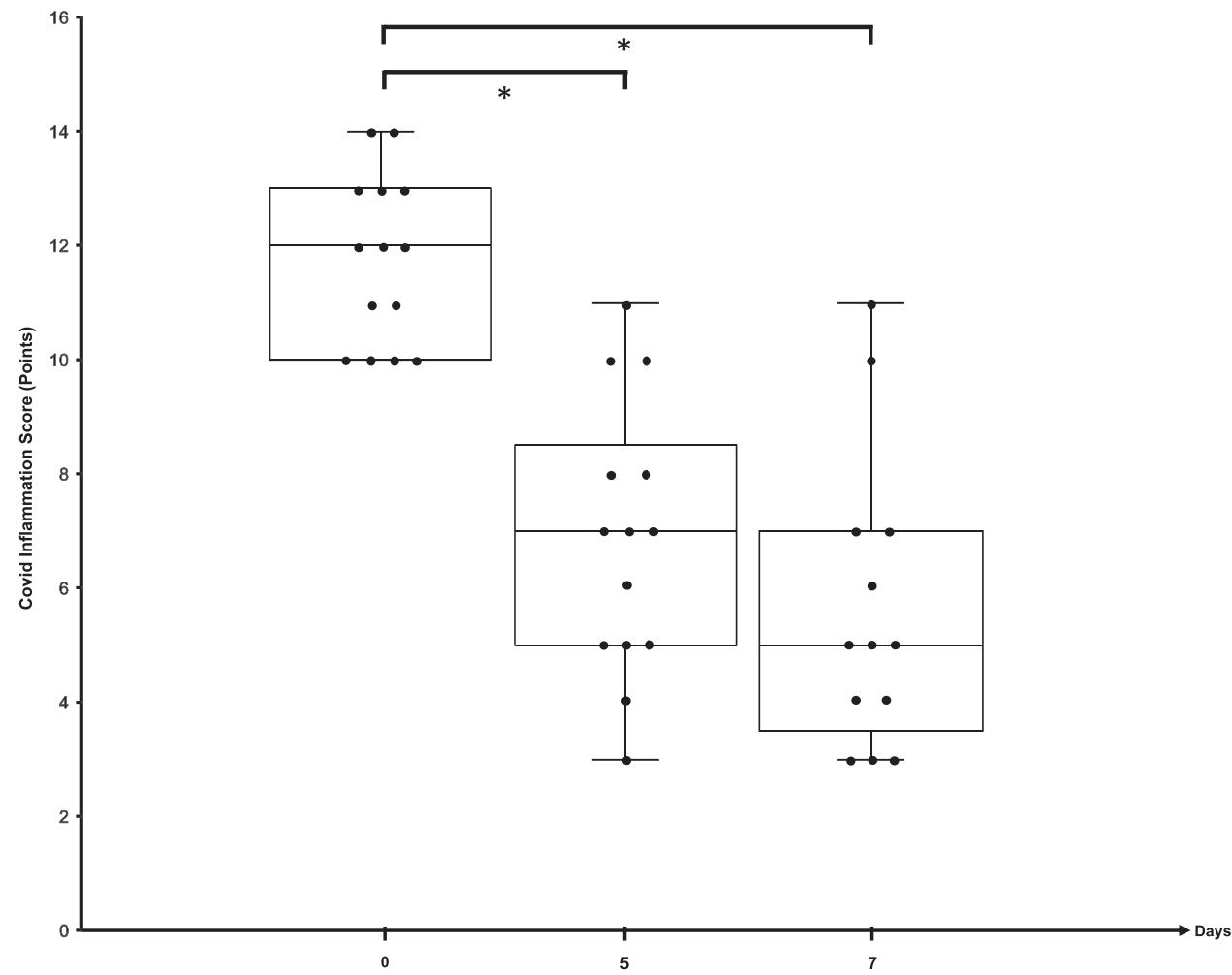

Table 5 Primary and secondary outcome results.

\begin{tabular}{|c|c|c|c|c|}
\hline & Total $(N=14)$ & & & \\
\hline CIS improvement Day $7>25 \%$, no. $(\%)^{\mathrm{a}}$ & $12(86)$ & & & \\
\hline Days of hospitalization, median (IQR) & $18(9-36)$ & & & \\
\hline Intermediate care, no. (\%) & $6(43)$ & & & \\
\hline Days of Intermediate care, median (IQR) & $5(2-12)$ & & & \\
\hline Intensive care, no. (\%) & $6(43)$ & & & \\
\hline \multirow[t]{2}{*}{ Days of Intensive care, median (IQR) } & $18.5(1-26)$ & & & \\
\hline & Baseline $(N=14)$ & Day $5(N=14)$ & Day $7(N=14)$ & Day $15(N=11)$ \\
\hline CIS improvement \%, median (IQR) & - & $42(15-70)$ & $58(15-77)^{\mathrm{b}}$ & $50(15-54)^{\mathrm{c}}$ \\
\hline NEWS2 scale, median (IQR) & $8.5(4-16)$ & $4.5(14-2)$ & $4(2-13)^{b}$ & $7(3-13)^{\mathrm{d}}$ \\
\hline \multicolumn{5}{|l|}{ 7-point ordinal scale, no. (\%) } \\
\hline 2-Not hospitalized, limitations on activities & $0(0)$ & $1(7)$ & $1(7)$ & $5(46)$ \\
\hline $\begin{array}{l}\text { 4-Hospitalized, requiring } \\
\text { supplemental oxygen }\end{array}$ & $2(14)$ & $0(0)$ & $3(21)$ & $1(9)$ \\
\hline $\begin{array}{l}\text { 5-Hospitalized, on non-invasive ventilation } \\
\text { or HFNC }\end{array}$ & $11(79)$ & $10(71)$ & $7(50)$ & $4(36)$ \\
\hline $\begin{array}{l}\text { 6- Hospitalization, requiring ECMO } \\
\text { invasive mechanical ventilation, or both }\end{array}$ & $1(7)$ & $3(21)$ & $3(21)$ & $1(9)$ \\
\hline
\end{tabular}

${ }^{\mathrm{a}} 13$ pts follow-up.

${ }^{\mathrm{b}} 13 \mathrm{pts}$ follow-up.

c 3 pts follow-up.

$\mathrm{d}_{4}$ pts follow-up.

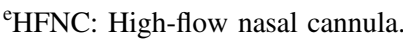

fECMO: Extracorporeal membrane oxygenation. 
Fig. 3 Suppression of inflammation by Rux in patients without concomitant corticosteroid treatment. ULN upper limit of normal.
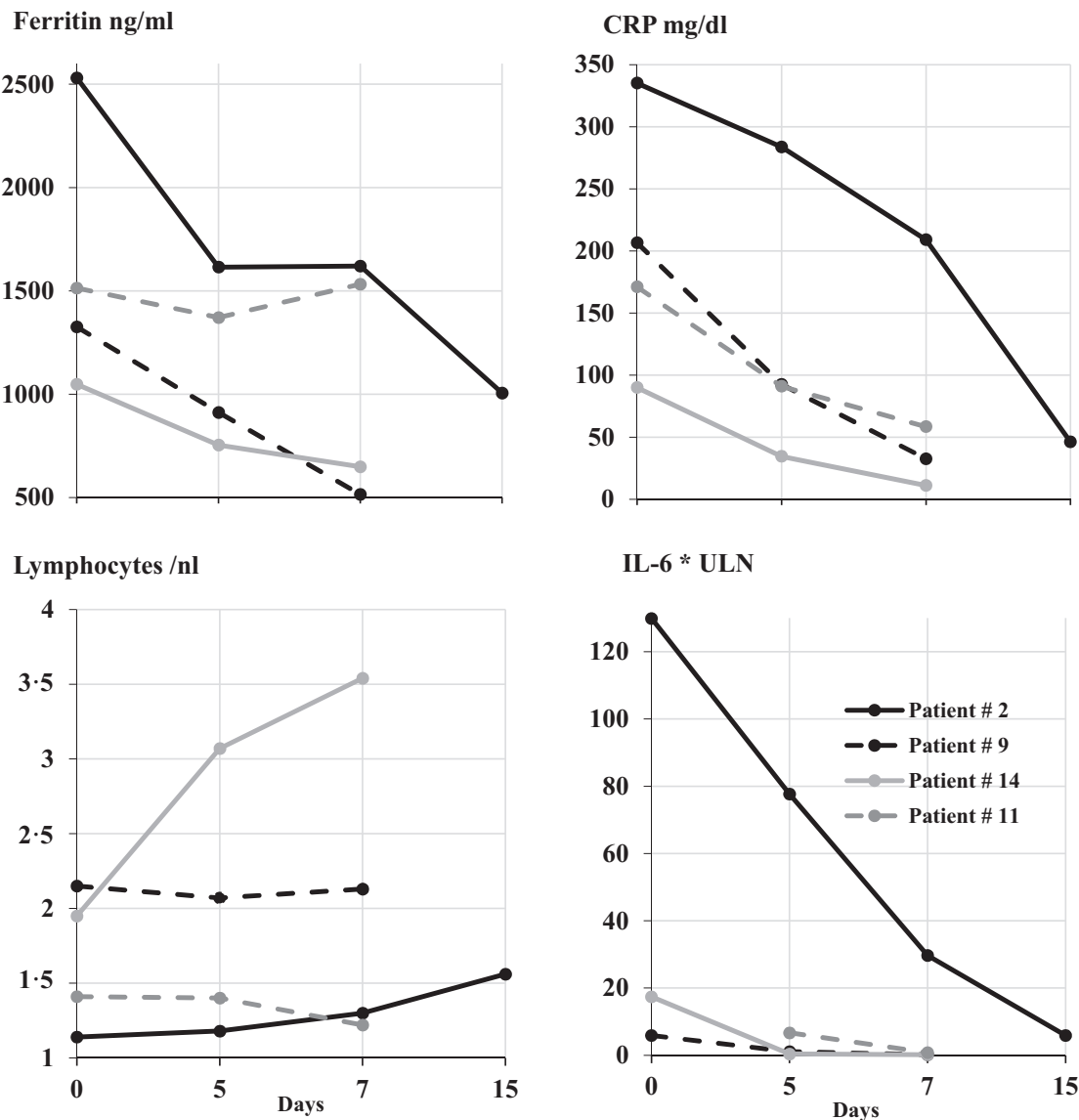

$$
\text { IL-6 * ULN }
$$

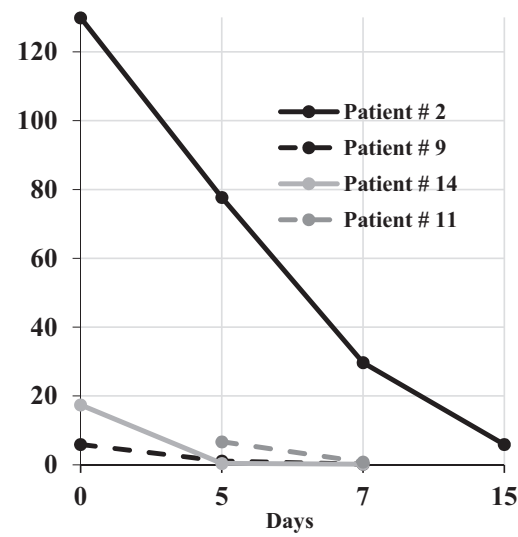

Table 4 by serial laboratory assessment, liver dysfunction as indicated by increased transaminases was moderate with one patient hitting transient grade 3 liver toxicity. Anemia grade 3 was seen in two patients with pre-existing anemia and treated on ICU with repetitive need for blood drawing. Viral clearance as assessed by soar swabs was checked for anecdotally with four patients tested negative for SARSCoV-2 while on or after Rux treatment (Fig. 4). One patient showed continuous SARS-CoV-2-positivity. She has been previously immunosuppressed and on renal replacement therapy for vasculitis. In summary, short term toxicity assessment using special interest side effects did not show red flag signals in this limited number of patients.

\section{Discussion}

COVID-19 is a RNA-virus-induced airborne infection with significant morbidity and mortality and no proven antiviral standard therapy. The current pandemic is a global health threat leading to collapse of health care systems even in the most developed countries due to bulk incidence of patients with respiratory failure. Vaccines are in development. Virus directed treatment is under investigation with preliminary efficacy signals in hitherto published trials [2, 3]. Combined antiviral/immunomodulatory treatment with hydroxychloroquine in some countries is a so to say standard with theoretical, but clinically not confirmed activity against COVID-19 [29, 30]. After the disaster of ICU over flooding patients in need of ventilators in Wuhan, Europe, or the USA, it became clear that a subfraction of severely affected patients show hyperactivated T- cells in the peripheral blood and the lungs with systemic hyperinflammation [4-7, 31] stimulating the hypothesis of virus-induced macrophage activation syndrome (MAS) or HLH as potential causes for the consecutive multiorgan failure $[19,20]$. Pointing into the same direction are considerations to specifically target the cytokine storm through anti-IL6 or anti-TNF directed antibodies [32-34]. We have not seen HLH/MAS-HLH in our series, as not a single patient showed bicytopenia nor extreme values for ferritin, two criteria with high HLHsensitivity [35]. We therefore argue for a note of caution against therapeutic actionism targeting classical HLH in COVID-19 using T-cell depleting etoposide [19].

We selected Rux for CRS-targeting due to its record in highly inflammatory conditions such as primary myelofibrosis, HLH, and GvHD [11, 12, 14]. The challenge of managing severely affected COVID-19 patients is the need to coordinate treatment between the COVID-ward medical team monitoring patients at risk for deterioration and to 


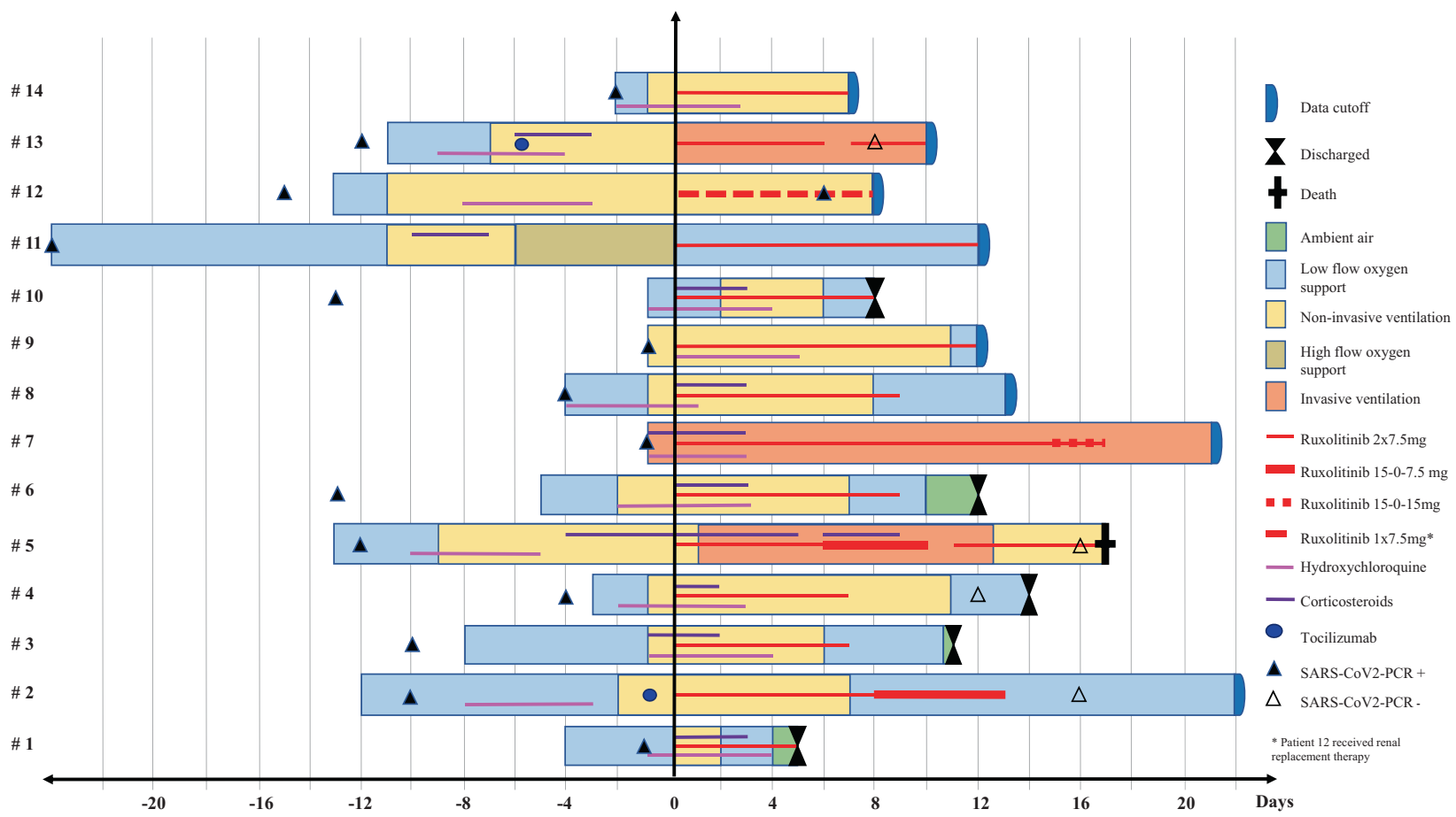

Fig. 4 Individual patient tracking with treatment applied and clinical course after Rux onset. Individual patients $(\mathrm{n}=14)$ are depicted on the $\mathrm{y}$-axis, treatment duration is shown on the $\mathrm{x}$-axis (days).

integrate subspeciality knowledge into ICU-management. In order to safeguard detection of patients at risk to develop a hyperinflammatory state, we developed the CIS as a predefined semiquantitative measure for CRS-directed treatment allocation with corticosteroids and/or Rux. This allowed us to develop a COVID-board learning curve for all team members in trying to differentiate COVID-specific inflammation from other causes of IL-6-driven inflammation (sepsis, superinfection). In an emergency treatment situation without available standard treatments and prior to activated clinical trial protocols, this interdisciplinary screening approach can enable medical teams to identify patients with a balanced risk-benefit ratio for novel treatment under investigation. Rux treatment was associated with meaningful inflammation control as assessed by CIS response on day 5 and day 7 in 12/14 pts that correlated with clinical response in 11 out of 14 patients (Fig. 1). Of note, this response was also shown to be achieved without concomitant CS treatment (4/14 pts) (Figs. 2 and 3). Devoid of systematic serial virus testing, we still have some indication of clinical recovery in 4 of the 14 patients with viral clearance despite the fact that Rux has been reported to have the potential of affecting virus control [28]. In this regard, experimental and clinical data suggest JAK1-dependent inhibition of T-cells by Rux explaining observed reactivation of atypical infections in patients on longterm ruxolitinib in MPN [18]. Our approach of short term Rux in severe Covid-19 is intented to stop detrimental cytokine signalling thereby preventing further organ damage. Unnecessary prolonged treatment was avoided to balance the need for calming the cytokine storm against the risk to provoke virus reactivation. Similarly, patients in virus-induced HLH are recommended not to receive extended duration of immunosuppression in order to allow viral clearance [36]. Side effects of short term Rux treatment are well manageable and show the expected spectrum with mild anemia and liver enzyme elevation.

Dosing without pre-existing evidence is a great matter of uncertainty. We developed our dosing guidelines along trials with Rux in GvHD with trial data suggesting good efficacy/toxicity relationship between $5 \mathrm{mg}$ and $10 \mathrm{mg}$ bid $[14,25]$, and in HLH with individual case reports and a phase I trial providing dose ranges between $5 \mathrm{mg}$ bid and $25 \mathrm{mg}$ bid [12, 13, 37]. Serial efficacy/toxicity assessment in the COVID-board was performed to trigger dose adaptation due to toxicity, or efficacy considerations. The results of treatment have led to the design of a clinical trial with a Rux dose of $10 \mathrm{mg}$ bid and potential dose increase up to 20 mg bid depending on CIS response on days 3, 5, and 7 (NCT04338958) based on the conclusion that our small case series provides us with surrogate and clinical efficacy in patients treated with $7.5 \mathrm{mg}$ bid, but failing in patients critically ill, deteriorating while on Rux treatment without strong signals of toxicity.

Several clinical trials are underway to test Rux in COVID19. Questions asked are: Is low dose (5 mg bid) compared to 
placebo efficacious in preventing moderately affected patients from progression to severe/critical disease stages (NCT04362137). Is Rux treatment able to show efficacy in severely affected patients on invasive ventilation due to ARDS (NCT04359290)? A Canadian trial using $10 \mathrm{mg}$ bid is addressing the potential cytokine flare [38] when taking patients off Rux from full dose to zero and directs tapered treatment discontinuation (NCT04331665).

The majority of COVID-19 patients show benign disease course where patients overcome viral inflammation by robust but not overreactive immune response [39]. Treating the majority of those could lead to significant overtreatment while achieving the goal of preventing patients from deteriorating into hyperinflammation. Treating patients on invasive ventilation for ARDS according to our series is likely to be most challenging as irreversible organ damage may be in place and secondary infections cross-react with IL-6/CRP-monitoring of Rux-response. This will require extended and probably dose increased treatment. Treatment in very advanced disease may fall short in preventing the ARDS-associated CRS from causing fatalities, yet this needs to be shown.

Rux is hypothesized to interfere with the detrimental CRS governed by pulmonary inflammation through interference with multiple pro-inflammatory cytokines via JAKSTAT-inhibition. In addition, it may act through antiviral activity by impairing viral replication through interaction with senescence regulation pathways [40]. We are currently in a period with highly vivid hypothesis driven, but low level evidence based clinical emergency management of COVID-19 patients due to lack of proven therapeutics. Our small pilot series has established a reproducible score (CIS) to stratify treatment, which will be tested in our recently initiated RuxCoFlam phase II trial (NCT04338958). To capture individual patients in need for tailored antiinflammatory treatment without putting too many of them at risk of unnecessary overtreatment, hospital wide education, standardized and interdisciplinary structured treatment and implementation of clinical trials seem to be pivotal.

Acknowledgements We gratefully acknowledge the support of the entire Covid-19 task force of the Schwarzwald-Baar-Klinikum, in particular nursing staff, respiratory specialists and many colleagues supporting the daily Covid-19 board. In particular thanks to Eike Walter, Albert Benzing, Werner Huber, Anja Rückert, Patricia Mohm, Stefanie Bergander, Robby Winkler, Kerstin Heine and Vanessa Kardinal. Patients and families for their sympathy and support. Open access funding provided by Projekt DEAL.

\section{Compliance with ethical standards}

Conflict of interest Nothing to disclose: FL, SB, HCB, MF, MH, BK, IG, AK, SR, Research support by Novartis, Incyte: AH, Adboard Novartis, Incyte: PL; Speakers office: PL.
Ethics This retrospective chart analysis was approved by the state's chamber of physician's review board (No. F-2020-052). It was performed according to the Declaration of Helsinki after informed consent.

Publisher's note Springer Nature remains neutral with regard to jurisdictional claims in published maps and institutional affiliations.

Open Access This article is licensed under a Creative Commons Attribution 4.0 International License, which permits use, sharing, adaptation, distribution and reproduction in any medium or format, as long as you give appropriate credit to the original author(s) and the source, provide a link to the Creative Commons license, and indicate if changes were made. The images or other third party material in this article are included in the article's Creative Commons license, unless indicated otherwise in a credit line to the material. If material is not included in the article's Creative Commons license and your intended use is not permitted by statutory regulation or exceeds the permitted use, you will need to obtain permission directly from the copyright holder. To view a copy of this license, visit http://creativecommons. org/licenses/by/4.0/.

\section{References}

1. Chen R, Liang W, Jiang M, Guan W, Zhan C, Wang T, et al. Risk factors of fatal outcome in hospitalized subjects with coronavirus disease 2019 from a nationwide analysis in China. Chest. 2020. https://doi.org/10.1016/j.chest.2020.04.010

2. Cao B, Wang Y, Wen D, Liu W, Wang J, Fan G, et al. A trial of Lopinavir-Ritonavir in adults hospitalized with severe covid-19. N Engl J Med. 2020. https://doi.org/10.1056/NEJMoa2001282.

3. Grein J, Ohmagari N, Shin D, Diaz G, Asperges E, Castagna A, et al. Compassionate use of remdesivir for patients with severe covid-19. N Engl J Med. 2020. https://doi.org/10.1056/NEJMoa 2007016

4. Huang C, Wang Y, Li X, Ren L, Zhao J, Hu Y, et al. Clinical features of patients infected with 2019 novel coronavirus in Wuhan, China. Lancet. 2020;395:497-506.

5. Zhou F, Yu T, Du R, Fan G, Liu Y, Liu Z, et al. Clinical course and risk factors for mortality of adult inpatients with COVID-19 in Wuhan, China: a retrospective cohort study. Lancet. 2020;395: 1054-62.

6. Song YG, Shin HS. COVID-19, a clinical syndrome manifesting as hypersensitivity pneumonitis. Infect Chemother. 2020;52: $110-2$.

7. Xu Z, Shi L, Wang Y, Zhang J, Huang L, Zhang C, et al. Pathological findings of COVID-19 associated with acute respiratory distress syndrome. Lancet Respir Med. 2020;8:420-2.

8. Ruan Q, Yang K, Wang W, Jiang L, Song J. Clinical predictors of mortality due to COVID-19 based on an analysis of data of 150 patients from Wuhan, China. Intens Care Med. 2020. https://doi. org/10.1007/s00134-020-05991-x

9. Yang Y, Shen C, Li J, Yuan J, Yang M, Wang F, et al. Exuberant elevation of IP-10, MCP-3 and IL-1ra during SARS-CoV-2 infection is associated with disease severity and fatal outcome. medRxiv. 2020: 2020.2003.2002.20029975.

10. Harrison C, Kiladjian JJ, Al-Ali HK, Gisslinger H, Waltzman R, Stalbovskaya V, et al. JAK inhibition with ruxolitinib versus best available therapy for myelofibrosis. N. Engl J Med. 2012;366: 787-98.

11. Lussana F, Rambaldi A. Inflammation and myeloproliferative neoplasms. J Autoimmun. 2017;85:58-63. 
12. Ahmed A, Merrill SA, Alsawah F, Bockenstedt P, Campagnaro E, Devata $\mathrm{S}$, et al. Ruxolitinib in adult patients with secondary haemophagocytic lymphohistiocytosis: an open-label, single-centre, pilot trial. Lancet Haematol. 2019;6:e630-e637.

13. Wang J, Wang Y, Wu L, Wang X, Jin Z, Gao Z, et al. Ruxolitinib for refractory/relapsed hemophagocytic lymphohistiocytosis. Haematologica. 2019. https://doi.org/10.3324/haematol.2019. 222471

14. Zeiser R, von Bubnoff N, Butler J, Mohty M, Niederwieser D, Or $\mathrm{R}$, et al. Ruxolitinib for glucocorticoid-refractory acute graftversus-host disease. N Engl J Med. 2020. https://doi.org/10.1056/ NEJMoa1917635

15. Zeiser R, Burchert A, Lengerke C, Verbeek M, Maas-Bauer K, Metzelder SK, et al. Ruxolitinib in corticosteroid-refractory graftversus-host disease after allogeneic stem cell transplantation: a multicenter survey. Leukemia. 2015;29:2062-8.

16. Wölfel R, Corman VM, Guggemos W, Seilmaier M, Zange S, Müller MA, et al. Virological assessment of hospitalized patients with COVID-2019. Nature. 2020. https://doi.org/10.1038/s41586020-2196-x

17. Caocci G, Murgia F, Podda L, Solinas A, Atzeni S, La Nasa G. Reactivation of hepatitis B virus infection following ruxolitinib treatment in a patient with myelofibrosis. Leuk: Off J Leuk Soc Am, Leuk Res Fund, UK. 2014;28(Jan):225-7.

18. Perner F, Schnoder TM, Ranjan S, Wolleschak D, Ebert C, Pils MC, et al. Specificity of JAK-kinase inhibition determines impact on human and murine T-cell function. Leuk: Off J Leuk Soc Am, Leuk Res Fund, UK. 2016;30:991-5.

19. Mehta P, McAuley DF, Brown M, Sanchez E, Tattersall RS, Manson JJ, et al. COVID-19: consider cytokine storm syndromes and immunosuppression. Lancet. 2020;395:1033-4.

20. Moore, BJB, June, CH. Cytokine release syndrome in severe COVID-19. Science. 2020. https://doi.org/10.1126/science.abb8925

21. Zhang W, Zhao Y, Zhang F, Wang Q, Li T, Liu Z, et al. The use of anti-inflammatory drugs in the treatment of people with severe coronavirus disease 2019 (COVID-19): the Perspectives of clinical immunologists from China. Clin Immunol. 2020;214:108393.

22. Cantini F, Niccoli L, Matarrese D, Nicastri E, Stobbione P, Goletti D. Baricitinib therapy in COVID-19: a pilot study on safety and clinical impact. J Inf. 2020. https://doi.org/10.1016/j.jinf. 2020.04.017

23. Wang $\mathrm{W}, \mathrm{Wu} \mathrm{S}$, Lie $\mathrm{P}$, Huang L, Li Y, Chen S, et al. The definition and risks of cytokine release syndrome-like in 11 COVID-19-infected pneumonia critically ill patients: disease characteristics and retrospective analysis. medRxiv. 2020. https:// doi.org/10.1101/2020.02.26.20026989

24. Wu Z, McGoogan JM. Characteristics of and important lessons from the coronavirus disease 2019 (COVID-19) outbreak in China: summary of a report of 72314 cases from the chinese center for disease control and prevention. Jama. 2020. https://doi. org/10.1001/jama.2020.2648

25. Jagasia M, Perales MA, Schroeder MA, Ali H, Shah NN, Chen YB, et al. Ruxolitinib for the treatment of steroid-refractory acute GVHD (REACH1): a multicenter, open-label, phase 2 trial. Blood. 2020. https://doi.org/10.1182/blood.2020004823

26. (RKI) RKI. Hinweise zu Erkennung, Diagnostik und Therapie von Patienten mit COVID-19. 2020. https://www.rki.de/DE/Content/
Kommissionen/Stakob/Stellungnahmen/Stellungnahme-Covid19_Therapie_Diagnose.pdf?_blob=publicationFile

27. Bhatraju PK, Ghassemieh BJ, Nichols M, Kim R, Jerome KR, Nalla AK, et al. Covid-19 in critically Ill patients in the seattle region - case series. N Engl J Med. 2020. https://doi.org/10.1056/ NEJMoa2004500

28. Sant'Antonio E, Bonifacio M, Breccia M, Rumi E. A journey through infectious risk associated with ruxolitinib. Br J Haematol. 2019;187:286-95.

29. Zhou D, Dai SM, Tong Q. COVID-19: a recommendation to examine the effect of hydroxychloroquine in preventing infection and progression. J Antimicrob Chemoth. 2020. https://doi.org/10. 1093/jac/dkaa114

30. Taccone FS, Gorham J, Vincent JL. Hydroxychloroquine in the management of critically ill patients with COVID-19: the need for an evidence base. Lancet Respir Med. 2020. https://doi.org/10. 1016/S2213-2600(20)30172-7

31. Chen G, Wu D, Guo W, Cao Y, Huang D, Wang H, et al. Clinical and immunological features of severe and moderate coronavirus disease 2019. J Clin Invest. 2020. https://doi.org/10.1172/ JCI137244

32. McGonagle D, Sharif K, O'Regan A, Bridgewood C. Interleukin6 use in COVID-19 pneumonia related macrophage activation syndrome. Autoim. Rev. 2020;19:102537.

33. Feldmann M, Maini RN, Woody JN, Holgate ST, Winter G, Rowland M, et al. Trials of anti-tumour necrosis factor therapy for COVID-19 are urgently needed. Lancet. 2020. https://doi.org/10. 1016/S0140-6736(20)30858-8

34. Xu X, Han M, Li T, Sun W, Wang D, Fu B, et al. Effective treatment of severe COVID-19 patients with tocilizumab. Proc Nat Acad Sci USA. 2020. https://doi.org/10.1073/pnas. 2005615117

35. La Rosée $\mathrm{P}$, Horne A, Hines $\mathrm{M}$, von Bahr Greenwood $\mathrm{T}$, Machowicz R, Berliner N, et al. Recommendations for the management of hemophagocytic lymphohistiocytosis in adults. Blood. 2019;133:2465-77.

36. Ehl S, Astigarraga I, von Bahr Greenwood T, Hines M, Horne A, Ishii E, et al. Recommendations for the use of etoposide-based therapy and bone marrow transplantation for the treatment of HLH: consensus statements by the HLH steering committee of the histiocyte society. J Allergy Clin Immunol Pr. 2018;6: 1508-17.

37. Zhao Y, Shi J, Li X, Wang J, Sun J, Zhou J, et al. Salvage therapy with dose-escalating ruxolitinib as a bridge to allogeneic stem cell transplantation for refractory hemophagocytic lymphohistiocytosis. Bone Marrow Transplant. 2019. https://doi.org/10.1038/ s41409-019-0577-2

38. Beauverd Y, Samii K. Acute respiratory distress syndrome in a patient with primary myelofibrosis after ruxolitinib treatment discontinuation. Int J Hematol. 2014;100:498-501.

39. Zhang Y. The epidemiological characteristics of an outbreak of 2019 novel coronavirus diseases (COVID-19) - China, 2020. China CDC Weekly; 2020.

40. Malavolta M, Giacconi R, Brunetti D, Provinciali M, Maggi F. Exploring the relevance of senotherapeutics for the current SARSCoV-2 emergency and similar future global health threats. Cells. 2020;9:909. https://doi.org/10.3390/cells9040909 